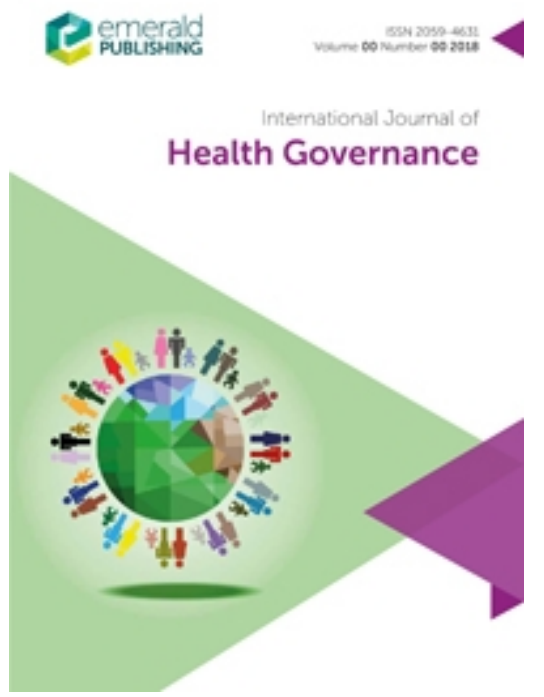

\title{
Research on hospital-based shared governance: a scoping review
}

\begin{tabular}{|r|l|}
\hline Journal: & International Journal of Health Governance \\
\hline Manuscript ID & ijhg-04-2020-0032 \\
\hline Manuscript Type: & Clinical Governance Reviews \\
\hline Keywords: & $\begin{array}{l}\text { Nursing < Health Professions, Clinical governance < Health Service } \\
\text { Quality Assurance, Continuous quality improvement < Health Service } \\
\text { Quality Assurance, Evidence-based practice < Health Service Quality } \\
\text { Assurance, Governance structures < Health Service Quality Assurance, } \\
\text { Health Professions }\end{array}$ \\
\hline
\end{tabular}

\section{SCHOLARONE \\ Manuscripts}




\section{Research on hospital-based shared governance: a scoping review}

\section{Purpose}

To review research on hospital-based shared governance, focusing on its core elements.

\section{Design}

A scoping review was conducted by searching the Medline (Ovid), CINAHL (EBSCO), Medic, ABI/INFORM Collection (ProQuest), and SveMed+ databases using SG and related concepts in hospital settings as search terms (May 1998-February 2019). Only original research articles examining SG were included. The reference lists of the selected articles were reviewed. Data were extracted from the selected articles by charting and then subjected to thematic analysis.

\section{Findings}

The review included 13 original research articles that examined SG in hospital settings. The studied organizations had implemented SG in different ways, and many struggled to obtain satisfactory results. SG was executed within individual professions or multiple professions and was typically implemented at both unit- and organization-levels. Thematic analysis revealed six core elements of SG: professionalism, shared decision-making, evidence-based practice (EBP), continuous quality improvement (CQI), collaboration, and empowerment.

\section{Practical implications}

An SG framework for hospital settings was developed based on the core elements of SG, the participants, and the organizational levels involved. Hospitals considering SG should prepare for a time-consuming process that requires belief in the core elements of SG. The SG framework can be used as a tool to implement and strengthen SG in organizations.

\section{Originality}

The review resumes the tradition of systematically reviewing SG literature, which had not been done in the $21^{\text {st }}$ century. General tendencies of the research scene and research gaps are pointed out.

\section{Key words}

Hospitals, Scoping review, Shared Governance, Nursing 


\section{BACKGROUND}

Shared governance (SG) is a structural phenomenon (Porter-O'Grady, 2001) that supports nurses' professional autonomy, control of their practice environment, and communication (Church et al., 2008). It originated in the USA in the late 1970s, when a nation-wide nursing shortage prompted healthcare organizations to reassess nurses' priorities (Porter-O'Grady, 2012). An organizational culture with an empowering approach to clinical and organizational decision making was considered central (Di Fiore et al., 2018) because the framework emerging from this reassessment aspired to change the structural reality in which nurses worked within formal, bureaucratic, and hierarchical organizations (Hess, 2004).

SG contributed to the creation of The Magnet Recognition Program ${ }^{\circledR}$, which aims to give hospitals tools to attract and retain qualified nurses. The program was initially available to American hospitals but was expanded internationally in 2000 (Morgan et al., 2006). A key objective in magnet hospitals is to achieve structural empowerment, for which SG provides a framework (Clavule et al., 2016).

In an SG organization, clinical practice decisions should be made by those who provide the care. The idea is that nurses own their practice environment and have a voice in decision-making (Winslow et al., 2015). Nurse managers have an important role as supporters of nurses in SG activities. The resulting partnership supports reciprocal communication, which is essential to the functioning of SG (Ott \& Ross, 2014).

Positive outcomes in nursing practice are achieved when nurses learn how to self-direct their work while aligning it with unit-level teams and nursing leadership (Joseph \& Bogue, 2016). Professional autonomy and decision-making skills can be enhanced by incorporating EBP into the SG framework and by enhancing staff nurses' leadership skills. Mentoring is one way of helping staff nurses become 
better clinical leaders (Dearmon et al., 2015). These changes take time, but eventually, staff nurses grow personally and professionally as they engage in SG activities (Guanxi, 2018).

Besides leadership skills, nurses need guidance on how to use research and conduct quality improvement processes to resolve system-wide practice issues (Dearmon et al., 2015). Many SG hospitals have engaged with this challenge by incorporating EBP into the SG councils' work methods (Fisher \& Hubbard, 2015) or by establishing an organization-wide EBP council (Gallagher-Ford, 2015).

Porter-O'Grady (1987) described three models of SG, namely the councillor (1), congressional (2), and administrative (3) models. The councillor model (1) facilitates SG through nursing councils, which make system-wide decisions relating to specified areas. The councils are overseen by an executive group that manages issues relating to the whole organization, coordinates the council's work, and integrates the council's work into practice. The congressional model (2) resembles a constitutional government: staff elect an executive and representatives to a nursing cabinet, which usually includes both managers and staff nurses. Finally, the administrative model (3) divides organizational authority between management and clinical forums that are overseen by an executive committee and a nurse executive (Porter-O'Grady, 1987). A fourth unit-based SG model under which SG is only implemented within units and not at the system level was recognized in the 1990s (Yanko et al. 1995).

Continuous evaluation of SG is recommended for successful human resource management and improvement of healthcare service quality (Gerard et al., 2016). The most widely used tool for assessing SG is “The Index of Professional Nursing Governance” (IPNG) (Hess, 1998; 2011), which measures six aspects of SG: professional control, organizational influence, organizational recognition, facilitating structures, liaison, and alignment. IPNG scores indicate which part of the SG 
range an organization occupies. The range extends from nursing management or administration only to staff nurses only, with the middle part of the range corresponding to a situation in which SG is equally distributed between staff nurses, nursing managers, and administrators (Hess, 1998). IPNG is considered the gold standard for surveying SG (Di Fiore et al., 2018).

Another way of evaluating SG is to measure structural empowerment in the organization (Joseph \& Bogue, 2016). Kanter's theory of empowerment suggests that in working life, access to information, support and resources, as well as opportunities to learn and grow, enable the empowerment of employees, which helps them accomplish organizational goals (Laschinger \& Wong, 1999). Structural empowerment is related to SG via elements such as autonomy, control over the practice environment, interprofessional collaboration, and access to information. It is also a contributing factor in enhancing magnet organizations and patient safety culture in healthcare (Armstrong \& Laschinger, 2006).

Other concepts related to SG are clinical and professional governance. Clinical governance is an umbrella term associated with attributes such as quality, continual improvement, standards of care, system-orientation, and responsibility (Brennan \& Flynn, 2013). It integrates activities targeting continuous quality improvement (Som, 2004). SG also shares this objective, although the SG literature places greater emphasis on empowering nurses than on SG's benefits to organizations (Porter-O'Grady, 2001; Hess, 2004). Professional governance refers to ideas of accountability, professional obligation, collateral relationships, and effective decision-making (Clavelle et al., 2016). It overlaps with SG via elements such as partnership (Hess, 2004), equity, and a strong emphasis on clinical nurses' decision-making (Porter-O’Grady, 2001).

The last systematic review to evaluate SG was made by O’May \& Buchan (1999). Since then, it has been stated that the gap between the concept of SG and its implementation requires study (Anthony, 
2004; Gerard et al., 2016) and that management strategies for implementing SG have not been standardized (Church et al., 2008). A need for an operational definition of SG (Joseph \& Bogue, 2016) and for robust assessments of the success of efforts to implement SG and their outcomes have also been noted (Anthony, 2004). Moreover, SG has evolved during its existence and continues to change. For example, in recent years, millennials have gained a strong footage in health care organisations, which has further accelerated the demands of renewing the structures and models that support professional nursing practice. (Porter-O’Grady, 2019.)

To conclude, this review is the first systematic review of SG written in the $21^{\text {st }}$ century and is needed because the concept of SG is broad and because its premises have changed over time. Since SG has mainly been implemented and studied in magnet hospitals, this scoping review focuses on hospitalbased SG. Its purpose is to provide an overview of research on hospital-based SG with a focus on core SG elements.

\section{METHODS}

A scoping review was conducted to explore and create an overview of the existing literature (Arksey \& O’Malley, 2005). The methodological frameworks for conducting scoping reviews presented by Levac et al. (2010) and Arksey \& O’Malley (2005) were adopted. To enhance the review's transparency and reporting quality, the "PRISMA Extension for Scoping Reviews" checklist was also used (Tricco et al., 2018). A three-step search strategy involving an initial search, a database search, and screening of the initially selected articles' reference lists was used to increase reliability.

Searches were performed using the following keyword combinations: SG (title) OR clinical governance (title) OR professional governance (title) AND model (abstract). The database search was conducted with the same search strategy in the five databases selected for consideration, yielding the 
following numbers of hits: Medline (Ovid) (100), CINAHL (EBSCO) (348), Medic (a Finnish database) (0), ABI/INFORM Collection (ProQuest) (360), and SveMed+ (a Swedish database) (0). The review included original research articles published in English, Finnish or Swedish. Because of the different ways in which SG can be implemented, only articles that described the applied SG model were eligible for inclusion. The beginning of the review's timespan (May 1998 - February 2019) corresponds to the end of the timespan of O’May \& Buchan's (1999) review of SG. Articles for which the full text was unavailable were excluded. In accordance with the criteria for scoping reviews, articles included in the review were not subjected to quality assessment (Armstrong et al. 2011).

The review process was conducted between February and March in 2019 by two independent researchers $(\mathrm{MK}, \mathrm{MH})$, first on the title and abstract level, then on the full-text level (Arksey \& O’Malley, 2005). After the selection process, a manual search was conducted by screening the reference lists of the selected articles (Armstrong et al., 2011). Refworks was used to facilitate the review process, including removal of duplicates. Eight original research articles were identified during the database search and five additional articles were identified by screening the reference lists of the initial eight articles (Figure 1).

The data were charted to identify common indicators of hospital-based SG and its core elements, as well as gaps in the literature (Armstrong et al. 2011). The charting categories correspond to extracted data relevant to the research objective. The categories are authors, year of publication, country, study design, participants, response rate, aims, methods used (instruments, statistical methods, and analytical methods), models, and key findings (Table 1).

Thematic analysis enables low-level interpretation and was therefore chosen to analyse the research articles (Vaismoradi et al., 2013). The analysis was conducted by one researcher (MK), but another researcher $(\mathrm{MH})$ was consulted in cases where the first researcher was uncertain. The analytical 
process involved first compiling the data and then separating and grouping it (Castleberry \& Nolen, 2018).

\section{RESULTS}

\section{Study characteristics}

All articles selected for inclusion in the review originated from the USA and were published between 2001 and 2018. The studies were mainly survey-based and used either longitudinal (Frith \& Montgomery, 2006; Brull, 2015; Allen-Gilliam et al., 2016; Ong et al., 2017; Di Fiore et al., 2018; Weaver et al., 2018;) or cross-sectional designs (Howell et al., 2001; Overcash et al., 2012; Rheingans, 2012; Wilson et al., 2014; Winslow et al., 2015; Gerard et al., 2016; Giambra et al., 2018). One study employed a modified Delphi technique (Giambra et al., 2018) and four studies utilized mixed methods (Frith \& Montgomery, 2006; Winslow et al., 2015; Ong et al., 2017; Giambra et al., 2018). The most widely used survey instrument was IPNG or its upgraded version (Howell et al., 2001; Overcash et al., 2012; Rheingans, 2012; Brull, 2015; Di Fiore et al., 2018; Weaver et al., 2018).

\section{Hospital-based SG models}

Three studies accepted participants other than nurses, such as nurse managers and patient care technicians (Frith \& Montgomery, 2006; Wilson et al., 2014; Giambra et al., 2018). In all of the studied settings, SG had originally been established for nurses, but two of the articles examined interdisciplinary models (Allen-Gilliam et al., 2016; Giambra et al., 2018). Studies on organizations that served only the nursing profession strongly emphasized nurse managers' supporting role. 
Most of the studies examined models implemented within the last 5 years (Howell et al., 2001; Frith \& Montgomery, 2006; Rheingans, 2012; Wilson et al., 2014; Brull, 2015; Ong et al., 2017; Di Fiore et al., 2018) or recently redesigned models (Winslow et al., 2015; Allen-Gilliam et al., 2016; Gerard et al., 2016; Giambra et al., 2018; Weaver et al., 2018). The models frequently had both a unit-level structure and an organization-wide structure (Frith \& Montgomery 2006; Rheingans, 2012; Wilson et al., 2014; Winslow et al., 2015; Brull, 2015; Gerard et al., 2016; Di Fiore et al., 2018; Giambra et al., 2018; Weaver et al., 2018). Only two articles described models consistent with Porter-O'Grady's (1987) original classification (Overcash et al., 2012; Rheingans, 2012). The articles described the SG models based on the levels at which SG was implemented (organization-wide, unit-based, or both) and the participants involved (single-profession or interdisciplinary).

Nine articles described the subjects addressed by the studied organizations' SG councils. Thematic analysis of these subjects revealed five council themes: education and research (Howell et al. 2001; Frith \& Montgomery 2006; Wilson et al. 2014; Brull 2015; Allen-Gilliam et al. 2016; Weaver et al. 2018), professional growth and development (Howell et al. 2001; Frith \& Montgomery 2006; Brull 2015; Winslow et al. 2015; Allen-Gilliam et al. 2016; Weaver et al. 2018), coordination and leadership (Howell et al. 2001; Frith \& Montgomery 2006; Winslow et al. 2015; Allen-Gilliam et al. 2016; Gerard et al. 2016; Di Fiore et al. 2018), safety and quality (Frith \& Montgomery 2006; Brull 2015; Allen-Gilliam et al. 2016; Weaver et al. 2018), and professional practice (Brull 2015; Winslow et al. 2015; Allen-Gilliam et al. 2016).

\section{Core elements}

Thematic analysis was used to identify the core elements of SG by first analysing the research themes of the articles and then categorizing these themes and using them to identify the core elements of SG. The first phase revealed five research themes - in order of decreasing frequency, the level of SG (1), 
barriers and facilitators of SG (2), the effect of demographic characteristics on nurses' views of SG (3), SG outcomes (4), and shared decision-making (5).

Most of the articles assessed the level of SG (1) using the IPNG, which emphasizes professionalism as a driver of SG. Few of the studied organizations' IPNG scores were in the SG range, indicating that most of the SG implementations were unsuccessful. Of the six organizations assessed using IPNG, only one exhibited a satisfactory level of SG without improving their SG model (Rheingans, 2012). Two other organizations achieved scores in the SG range in the third measurement round after redesigning their SG model (Brull, 2015; Weaver et al., 2018).

One of these organizations redesigned its SG model based on the wishes of staff, which included more education on SG, better communication and unit-based council meetings, involvement of night shift nurses, and increased participation of nurses in SG activities (Weaver et al., 2018). The second organization also aimed to increase nurses' participation in decision-making at the organization level and added more councils (Brull, 2015). The other studied organizations were perceived to be traditionally governed or led mainly by nursing management (Howell et al., 2001; Overcash et al., 2012; Di Fiore et al., 2018). Some studies found that active involvement in SG predicts more positive views of SG across organizational levels (Frith \& Montgomery, 2006; Overcash et al., 2012; Rheingans, 2012).

Three studies investigated the barriers and facilitators of SG (2). Barriers included disruption of patient care (Wilson et al., 2014) and an information deficit (Giambra et al., 2018). Facilitators included manager support and time to participate in SG activities (Frith \& Montgomery, 2006; Wilson et al., 2014; Giambra et al., 2018). Some studies also investigated the effects of nurses' demographic characteristics (e.g. work experience and education) on their view of SG (3) (Howell et al. 2001; Frith \& Montgomery 2006; Overcash et al. 2012; Rheingans 2012; Brull 2015). However, the observed 
correlations between demographic characteristics and views on SG were either non-significant or inconsistent.

Three articles assessed outcomes of SG (4) including self-perceived caring, the safety climate, and job satisfaction (Rheingans, 2012); the professional practice environment, empowerment, teamwork, risk of practice errors, and EBP (Allen-Gilliam et al., 2016); and engagement and turnover (Ong et al. 2017). Rheingans (2012) examined a councillor model that achieved positive outcomes, but the organization-wide model studied by Allen-Gilliam et al. (2016) and the unit-based model studied by Ong et al. (2017) both had inconclusive outcomes.

Shared decision-making (5) was analysed in three studies and addressed in four (Brull 2015; AllenGilliam et al., 2016; Giambra et al., 2018; Weaver et al., 2018). Winslow et al. (2015) found that once an SG model was implemented and became part of the organizational culture, shared decisionmaking grew stronger. Gerard et al. (2016) found that nurses in the organization desired more decision-making capacity. Similarly, Di Fiore et al. (2018) concluded that nurses' assessments of organizational decision-making were less positive than would be desirable.

CQI and EBP were not major research themes of any of the studies. However, EBP served as a framework in one study that evaluated the implementation of SG (Ong et al., 2017). It was also an explicit factor of the SG models considered in four articles (Brull, 2015; Winslow et al., 2015; AllenGilliam et al., 2016; Di Fiore et al., 2018). CQI was identified as an objective of the organization's SG model in five articles (Howell et al., 2001; Rheingans, 2012; Wilson et al., 2014; Allen-Gilliam et al., 2016; Ong et al., 2017). It was also a council theme in four organizations (Frith \& Montgomery, 2006; Brull, 2015; Di Fiore et al., 2018; Weaver et al., 2018). 
Collaboration was another element that was not used as a research theme. However, many of the articles highlighted collaboration between professionals (Howell et al., 2001; Wilson et al., 2014), between management and staff (Wilson et al., 2014; Gerard et al., 2016; Ong et al., 2017), and between organizational levels (Rheingans 2012; Ong et al., 2017) as core elements of successful SG.

Empowerment was mentioned as a main interest in one study (Allen-Gilliam et al., 2016). However, when the articles were analysed through Kanter's theory of empowerment, the council themes clearly included aspects of empowerment such as professional growth and development as well as professional practice, which highlight professionalism and the work force's opportunities for growth (Howell et al. 2001; Frith \& Montgomery 2006; Brull 2015; Winslow et al. 2015; Allen-Gilliam et al. 2016; Weaver et al. 2018). Other overlapping themes included education, research, and EBP, which can all be linked to empowerment through access to information and learning.

The analysis thus revealed six core elements of SG: professionalism, shared decision-making, EBP, CQI, collaboration, and empowerment. Of these elements, professionalism is a string that ties everything together. Shared decision-making is an element that allows SG to manifest itself, while EBP provides a tool for decision-making. CQI is the objective of SG. The element of collaboration then represents the values embedded in the SG phenomenon - the idea of working together towards shared goals. Empowerment is thus an underlying element that gives momentum to SG.

To help organizations establish SG, a framework based on the main findings of this review was created by combining the structural choices and core elements identified above to provide an overview of SG in hospital settings. The framework is presented in the form of a map of outcomes showing the frequency at which each SG factor was discussed in the reviewed articles (Figure 2). 


\section{DISCUSSION}

This scoping review resumes the tradition of systematically reviewing SG literature, which had not been done in the $21^{\text {st }}$ century (O’May \& Buchan 1999). The aim was to review research on hospitalbased shared governance, focusing on its core elements. Thematic analysis of the reviewed articles suggests that the core elements of SG in hospital settings are professionalism, shared decisionmaking, EBP, CQI, collaboration, and empowerment. These core elements are equivalent to those recognized by previous researchers (O’May \& Buchan, 1999; Porter-O'Grady, 2001; Anthony, 2004; Hess, 2011), except that CQI and EBP have not previously been linked so directly to SG. The two new elements reflect the changing healthcare environment, which has placed a greater emphasis on patients' rights to first-rate care.

SG is executed in varying ways, as noted previously by Church et al. (2008) and Joseph \& Bogue (2016). Based on the results obtained here, organizations do not seem to strive for a standardized way of implementing SG because only two articles described models consistent with Porter-O'Grady's (1987) classification. It is thus possible that this classification does not serve the current needs of the healthcare arena. Since this first classification, healthcare organizations and disciplines have evolved, and especially in the nursing field, professional development has changed the way in which the borders of competences, decision-making, and autonomy are defined (Fisher \& Hubbard, 2015; Gallagher-Ford, 2015).

This review endorses the research articles' approach of classifying SG models more simply, as being either organization-wide or unit-based, and as being executed within a single profession or an interdisciplinary group. This classification augments organizations' ability to define themselves in relation to other SG organizations while leaving space for innovation. SG can be implemented at different levels depending on organizational priorities (Gallagher-Ford, 2015; Gerard et al., 2016). 
Team characteristics are also important; for example, the implementation of SG in a highly experienced ICU nursing team may differ from that in a team consisting mainly of novice nurses.

\title{
Study limitations
}

The scoping review has several limitations. Firstly, only the most relevant databases were selected as information retrieval sources. Two databases in languages other than English were also included to obtain an overview of SG research outside English-speaking countries. Secondly, the review only included articles that examined SG in hospital settings. Thirdly, the review included only original research articles. Other types of articles were excluded because of their variable quality. The review's validity was strengthened by consulting a librarian when planning the search strategy. Additionally, the search was conducted by two independent researchers, first on the title and abstract level and then on the full-text-level. The analysis was then discussed between researchers to add validity.

\begin{abstract}
All the included research articles deal with SG even though professional and clinical governance were also used as search terms, which might be due to differences in execution. It should also be noted that the actual database screening identified eight relevant articles, whereas screening of the included articles' reference lists revealed five relevant articles. All the studies included in the review were conducted in the USA, possibly due to a language bias. The Finnish and the Swedish databases yielded no relevant articles. Additionally, the magnet hospital movement, which has favoured SG, has been largely restricted to the USA.
\end{abstract}

\section{CONCLUSIONS}


Six core elements of SG were identified in this review: professionalism, shared decision-making, EBP, CQI, collaboration and empowerment. The findings of this review are summarized in a new SG framework consisting of these core elements as well as the participants in the SG process and the organizational levels at which SG is implemented. Additionally, our analysis endorses a classification of SG in hospital settings that is simpler than that of Porter-O'Grady (1987), with SG organizations being classified based on the professionals included in SG activities (single profession and managers only or interdisciplinary) and the level at which SG activities are conducted (unit-based, organizationwide, or both).

The core elements should be monitored to keep up with changes in the healthcare arena. Further systematic reviews should be performed periodically to accumulate data on SG, which continues to inspire organizations, professionals, and researchers despite the challenges they face. Since many organizations found it difficult to achieve a satisfactory level of SG, continued assessment of SG levels is recommended, along with further exploration of barriers and facilitators of SG. There is also a notable lack of research on the effects of SG on patients, which should be addressed.

\section{PRACTICAL IMPLICATIONS}

Based on the findings of this review, an updated SG framework has been developed. This framework could be used to guide and strengthen the implementation of SG and the updated core elements should be considered in SG organizations. Additionally, the benefits of SG to organizations and patients as well as professionals should be emphasized. By combining EBP and SG, knowledge and knowhow can be transferred from professionals to the whole organization through the SG structure, ensuring that improvements are not dependent on the presence of specific individuals. 
The implementation of SG is not always as straightforward as first thought because even more mature SG organizations face challenges in meeting the expectations of the workforce. This review shows that SG can be achieved, but not without meaningful effort from the facility and its workforce. In other words, SG is not for the impatient looking for an easy fix, but for those who truly want to be part of an organization that gives a voice to its professionals. 


\section{References}

Allen-Gilliam, J., Kring, D., Graham, R., Freeman, K., Swain, S., Faircloth, G., and Jenkinson, B. (2016), "The impact of shared governance over time in a small community hospital", Journal of Nursing Administration, Vol. 46 No. 5, pp. 257-264.

Anthony, M. K. (2004), "Shared governance models: The theory, practice, and evidence", Online Journal of Issues in Nursing, Vol. 22 No. 1, pp. 138-153.

Arksey, H., and O'Malley, L. (2005), "Scoping studies: Towards a methodological framework", International Journal of Social Research Methodology, Vol. 8 No. 1, pp. 19-32.

Armstrong, R., Hall, B. J., Doyle, J., and Waters, E. (2011), “Cochrane update. 'Scoping the scope' of a cochrane review", Journal of Public Health (Oxford, England), Vol. 33 No. 1, pp. 147-150.

Armstrong, K. J., and Laschinger, H. (2006), "Structural empowerment, Magnet hospital characteristics, and patient safety culture: making the link", Journal of Nursing Care Quality, Vol. 21 No. 2, pp. 124-132.

Brennan, N. M., and Flynn, M. A. (2013), "Differentiating clinical governance, clinical management and clinical practice", Clinical Governance, Vol. 18 No. 2, pp. 114-131.

Brull, S. (2015), "Successful shared governance through education", Nursing Economics, Vol. 33 No. 6, pp. 314-319.

Castleberry, A., and Nolen, A. (2018), "Thematic analysis of qualitative research data: Is it as easy as it sounds?" Currents in Pharmacy Teaching and Learning, Vol. 10, pp. 807-815.

Church, J. A., Baker, P., and Berry, D. M. (2008), "Shared governance: A journey with continual mile markers", Nursing Management, Vol. 39 No. 4, pp. 34-38.

Clavelle, J. T., O'Grady, T. P., and Drenkard, K. (2013), "Structural empowerment and the nursing practice environment in magnet ${ }^{\circledR}$ organizations." Journal of Nursing Administration, Vol. 43 No. 11 , pp. 566-573.

Dearmon, V., Riley, B., Mestas, L., and Buckner, E. (2015), "Bridge to shared governance:

Developing leadership of frontline nurses”, Nursing Administration Quarterly, Vol. 39 No. 1, pp. 69-77.

Di Fiore, T., Zito, A., Berardinelli, A., Bena, J. F., Morrison, S. L., Keck, D. E., . . Albert, N. M. (2018), "Staff perceptions of decision-making in a shared governance culture", Journal of Nursing Administration, Vol. 48 No. 11, pp. 561-566.

Fisher, C., and Hubbard, M. (2015), "Extending evidence through shared governance”, Nursing Management, Vol. 46 No. 7, pp. 16-18.

Frith, K., and Montgomery, M. (2006), "Perceptions, knowledge, and commitment of clinical staff to shared governance", Nursing Administration Quarterly, Vol. 30 No. 3, pp. 273-284.

Gallagher-Ford, L. (2015), "Leveraging shared governance councils to advance evidence-based practice: The EBP council journey", Worldviews on Evidence-Based Nursing, Vol. 12 No. 1, pp. 61-63. 
Gerard, S., Owens, D., and Oliver, P. (2016), "Nurses' perception of shared decision-making processes: Quantifying a shared governance culture", Journal of Nursing Administration, Vol. 46 No. 9, pp. 477-483.

Giambra, B., Kneflin, N., Morath, H., Lee, J., Lin, L., and Morris, E. (2018), “Meaningful Participation and Effective Communication in Shared Governance", Nurse Leader, Vol. 16 No. 1, pp. 48-53.

Guanci, G. (2018), “The nurse manager's role in a shared governance culture”, Nursing Management, Vol. 49 No. 6, pp. 46-50.

Hess, R. (1998), “Measuring nursing governance.” Nursing Research, Vol. 47 No. 1, pp. 35-42.

Hess, R. (2004), "From bedside to boardroom - nursing shared governance", Online Journal of Issues in Nursing, Vol. 9 No. 1.

Hess, R. (2011), "Slicing and dicing shared governance: In and around the numbers", Nursing Administration Quarterly, Vol. 35 No. 3, pp. 235-241.

Howell, J., Frederick, J., Olinger, B., Leftridge, D., Bell, T., Hess, R., and Clipp, E. (2001), “Can nurses govern in a government agency?” Journal of Nursing Administration, Vol. 31 No. 4, pp. 187-195.

Joseph, L., and Bogue, R. (2016), "A theory-based approach to nursing shared governance", Nursing Outlook, Vol. 64 No. 4, pp. 339-351.

Laschinger, H., and Wong, C. (1999), "Staff nurse empowerment and collective accountability: effect on perceived productivity and self-rated work effectiveness", Nursing Economic\$, Vol. 17 No. 6, pp. 308-351.

Levac, D., Colquhoun, H., and O'Brien, K. (2010), "Scoping studies: Advancing the methodology", Implementation Science, Vol. 5 No. 69, pp. 1-9.

Morgan, S., Lahman, E., and Hagstrom, C. (2006), “The Magnet Recognition Program ${ }^{\circledR}$ : Transforming Healthcare Through Excellence in Nursing Services", Journal of Nursing Care Quality, Vol. 21 No. 2, pp. 119-120.

O'May, F., and Buchan, J. (1999). Shared governance: A literature review. International Journal of Nursing Studies, 36(4), 281-300.

Ong, A. (2017), "Ripple effect: Shared governance and nurse engagement", Nursing Management, Vol. 48 No. 10, pp. 28-34.

Ott, J., and Ross, C. (2014), "The journey toward shared governance: The lived experience of nurse managers and staff nurses", Journal of Nursing Management, Vol. 22 No. 6, pp. 761-768.

Overcash, J., Petty, L. J., and Brown, S. (2012), "Perceptions of shared governance among nurses at a midwestern hospital”, Nursing Administration Quarterly, Vol. 36 No. 4, pp. 1-11.

Porter-O’Grady T (1987), “Shared Governance and New Organizational Models”, Nursing Economic\$, Vol. 5 No. 6, pp. 281-286.

Porter-O'Grady, T. (2001), “Is shared governance still relevant?”, Journal of Nursing Administration, Vol. 31 No. 10, pp. 468-473.

Porter-O'Grady, T. (2012), "Reframing knowledge work: Shared governance in the postdigital age", Creative Nursing, Vol. 18 No. 4, pp. 152-159. 
Porter-O'Grady, T. (2019), "Principles for sustaining shared/ professional governance in nursing", Nursing Management, Vol. 50 No. 1, pp. 36-41.

Rheingans, J. I. (2012), "The alchemy of shared governance: Turning steel (and sweat) into gold", Nurse Leader, Vol. 10 No. 1, pp. 40-42.

Som, C. (2004), "Clinical governance: a fresh look at its definition. Clinical Governance: An International Journal”, Vol. 9 No. 2, pp. 87-90.

Tricco, A., Lillie, E., Zarin, W., O'Brien, K., Colquhoun, H., Levac, D., . . Straus, S. (2018), "PRISMA extension for scoping reviews (PRISMA-ScR): Checklist and explanation", Annals of Internal Medicine, Vol. 169 No. 7, pp. 1-6.

Yanko, J. R., Hardt, M., and Bradstock, J. (1995), “The clinical nurse specialist role in shared governance", Critical Care Nursing Quarterly, Vol. 18 No. 3, pp. 87-90.

Vaismoradi, M., Turunen, H., and Bondas, T. (2013), "Content analysis and thematic analysis: Implications for conducting a qualitative descriptive study", Nursing and Health Sciences, Vol. 15 No. 3, pp. 398-405.

Weaver, S. H. (2018), "Measuring shared governance: One healthcare system's experience”, Nursing Management, Vol. 49 No. 10, pp. 11-14.

Wilson, J., Gabel Speroni, K., Jones, R. A., and Daniel, M. G. (2014), "Exploring how nurses and managers perceive shared governance", Nursing, Vol. 44 No. 7, pp. 19-22.

Winslow, S., Hougan, A., Deguzman, P., and Black, A. (2015), “The voice of the nurse... What's being said about shared governance?”, Nursing Management, Vol. 46 No. 3, pp. 46-51. 


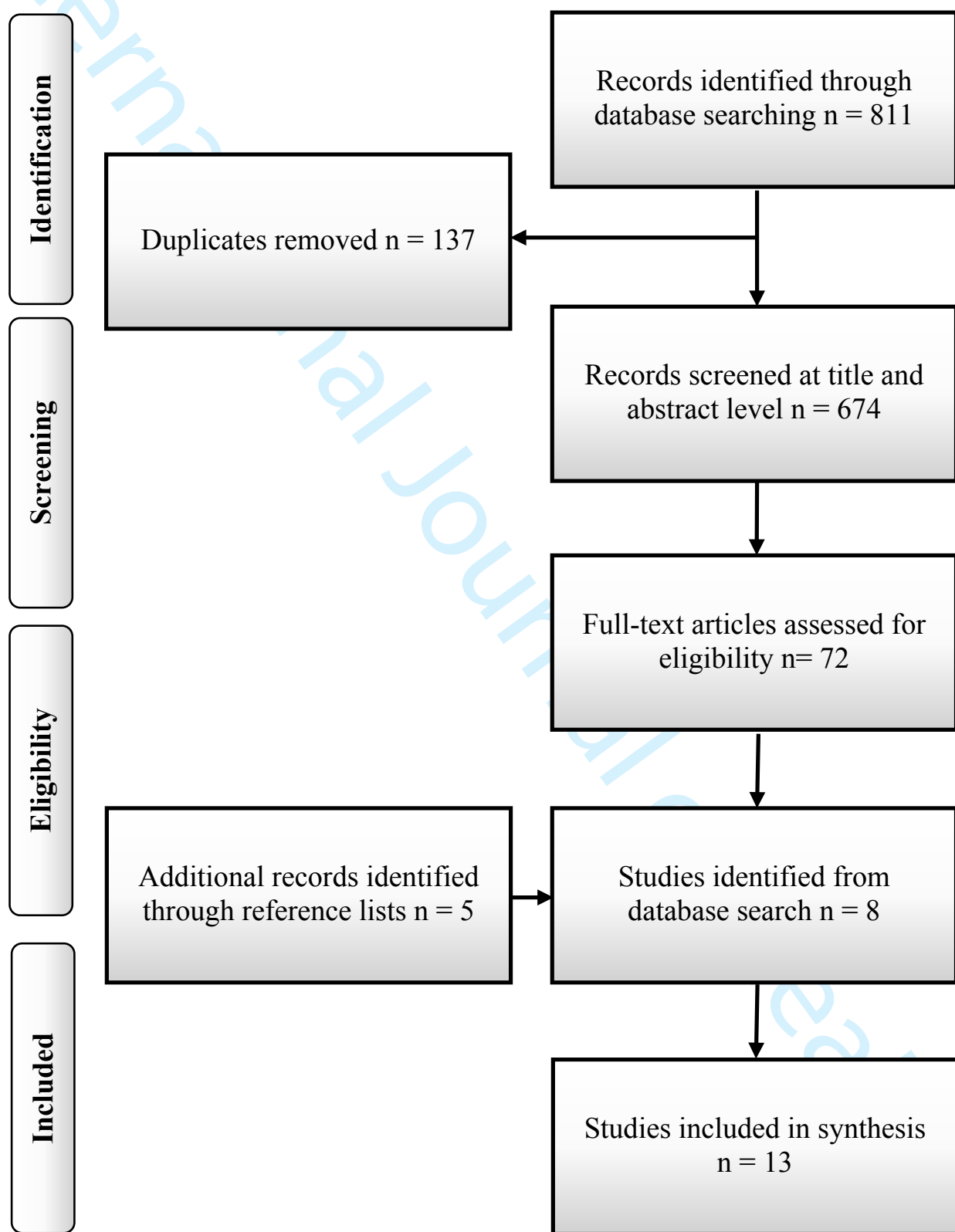

Records excluded $\mathrm{n}=$ 602

Full-text articles excluded, with reasons $\mathrm{n}=64$

not an original article: 49

full-text unavailable: 10 model not described: 3 not hospital-based: 2

Figure 1. Flowchart of the article selection process. Adapted from Moher et al. (2009). 
Table 1. Data extracted from the research articles.

\begin{tabular}{|c|c|c|c|c|}
\hline $\begin{array}{l}\text { Author(s), } \\
\text { year of } \\
\text { publication, } \\
\text { country }\end{array}$ & $\begin{array}{l}\text { Design, participants, Aims } \\
\text { response rate (rr) }\end{array}$ & $\begin{array}{l}\text { Methods } \\
\text { (instruments, } \\
\text { statistical } \\
\text { methods, } \\
\text { methods of } \\
\text { analysis) }\end{array}$ & SG model & Key findings \\
\hline
\end{tabular}

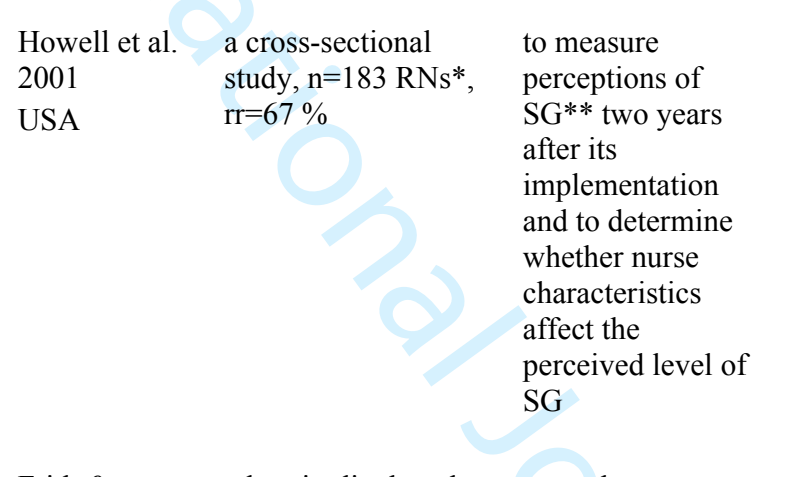

Frith \& a longitudinal study, to explore

Montgomery $\mathrm{n}=687 \mathrm{RNs}, \quad$ perceptions and

a survey

utilizing

$\mathrm{IPNG}^{* * *}$ and

organizatio the organization is

statistical

n-wide perceived to be

analysis,

including

descriptive

statistics and

measures of

central tendency

councils traditionally

governed;

nurse

demographics do

not significantly

correlate with

IPNG scores

2006

licensed practical

knowledge of SG

as well as

technicians, and

medical

commitment to

SG before and

receptionists, $\mathrm{rr}=33$

$\%$

after its

a survey

utilizing the

Shared

Governance

Survey adapted

from Minors et

al.,

second round:

$\mathrm{n}=961, \mathrm{rr}=48 \%$

focus group

implementation

statistical

analysis,

including

descriptive

statistics

interviews, $\mathrm{n}=68$

clinical and

managerial staff

Rheingans a cross-sectional

to determine study, $\mathrm{n}=140$ nurses

whether SG is

present in the

organization five

years after its

implementation

and to investigate

if SG resulted in

improvements

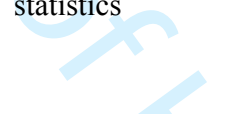

a survey

utilizing IPNG,

the Caring

Nurse-Patient

Interactions

Scale, the

Measure of Job

Satisfaction and

the Safety

Climate Survey

as instruments,

statistical

analysis,

including

regression

analyses

$\begin{array}{ll}\text { Overcash et } & \text { a cross-sectional } \\ \text { al. } 2012 & \text { study, } \mathrm{n}=100 \mathrm{RNs} \\ \text { USA } & \end{array}$

to determine

whether

demographic

variables affect

nurses' level of

perceived SG a survey

utilizing IPNG

and

statistical

analysis,

including

analysis of unit level implementation of

and SG is time-

organizatio consuming, but

n-wide staff remain

councils committed to it;

facilitating factors

of SG include

management

support, providing

time for council

work and

educating council

members about

SG;

monthly council

meetings and

greater than $50 \%$

attendance predict

more positive

views

IPNG score is in

$\begin{array}{ll}\text { councillor } & \text { the SG range; } \\ \text { model": } & \text { work status, }\end{array}$

unit level hospital unit,

and participation in

organizatio hospital-wide

n-wide councils, and

councils professional

certification affect

nurses'

evaluations organizatio

n-wide

councils the governance

model is

perceived to be

primarily led by

nursing

management

/administration 
variance and regression models as well as general linear models

to determine which factors affect SG participation and whether there are differences between staff nurses' and nurse managers' perceptions

$\begin{array}{ll}\text { Winslow et } & \text { a cross-sectional } \\ \text { al. } 2015 & \text { study, } \mathrm{n}=113 \mathrm{RNs}, \\ \text { USA } & \mathrm{rr}=25 \%\end{array}$

to determine whether the SG model meets the needs of shared decision-making after organizational changes and to measure nurses' understanding of SG

a longitudinal study,
$\mathrm{n}=88$ nurses,
$\mathrm{rr}=34 \%$
second round:
$\mathrm{n}=140, \mathrm{rr}=50 \%$
third round: $\mathrm{n}=107$,
$\mathrm{rr}=41 \%$

Brull 2015

USA

to determine whether an education plan strengthened SG by assessing changes in the level of SG, and to evaluate the impact of demographic variables on $\mathrm{SG}$ scores a survey
utilizing a 26-
item instrument
created for the study,

statistical

analysis,

including

frequency

distributions,

Chi-square

analysis and

Fisher's exact

tests

a survey

utilizing an

instrument

developed for the study,

thematic and

statistical

analysis,

including

calculation of

means and the

Mann-Whitney

$\mathrm{U}$ test

a survey
utilizing IPNG
and
statistical
analysis,
including
frequency
distributions and
variance analysis

with some staff nurse input;

having a role in

SG and working

in in-patient

settings affects

evaluation

$\begin{array}{ll}\text { unit level } & \text { staff nurses' } \\ \text { and } & \text { participation in } \\ \text { organizatio } & \text { SG is increased } \\ \text { n-wide } & \text { when they work as } \\ \text { councils } & \text { a team, are } \\ & \text { supported by } \\ & \text { nurse managers, } \\ & \text { get compensation } \\ & \text { for participating, } \\ & \text { and participation } \\ & \text { does not disrupt } \\ & \text { patient care }\end{array}$

unit level $\quad 77 \%$ believed SG

and gives nurses a

organizatio voice in decision-

n-wide making, $80 \%$

councils agreed they had

adequate

resources and

encouragement to

make council

work meaningful;

qualitative

themes: SG is a

pathway for

nurses to have a

strong voice, it is

based on

ownership of the

practice

environment and

various disciplines

identifying issues

and collaborating

to identify

solutions

unit level a strategic

and approach to SG

organizatio implementation

n-wide had a significant

councils effect;

the baseline IPNG

score indicated a

traditionally

governed

organization but

the score

increased

marginally after

one year and

entered the SG

range after two

years 
Allen-

Gilliam et al.

2016

USA

a longitudinal study, $\mathrm{n}=52-142 \mathrm{RNs}$ and

licenced practical

nurses, $\mathrm{rr}=24-65 \%$

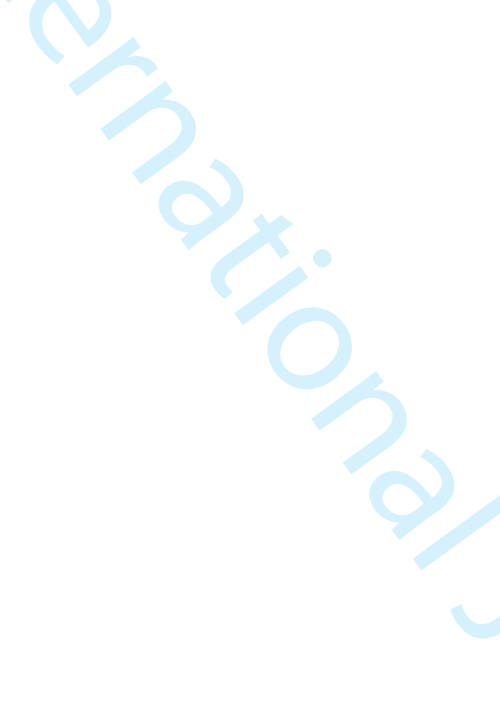

Gerard et al

a cross-sectional

2016

USA

$$
\text { study, } n=162 \text { staff }
$$
nurses, $\mathrm{rr}=21 \%$

Ong et al.

a longitudinal study,

2017

USA

$\mathrm{n}=19 \mathrm{RNs}, \mathrm{rr}=17 \%$

two focus groups

Giambra et al. 2018

USA to measure the

impact of a

redesigned $\mathrm{SG}$

model on the

professional

nursing practice

environment

(nursing

leadership, nurse

empowerment,

nurse satisfaction,

risk of practice

errors, and

comfort with

EBP) at baseline

and in subsequent

years

to describe how

measuring the

perceived and

desired decision-

making capacity

of nurses working

in a SG

organization can

be beneficial

to evaluate the

newly

implemented SG

model's impact on

RNs' engagement

and turnover

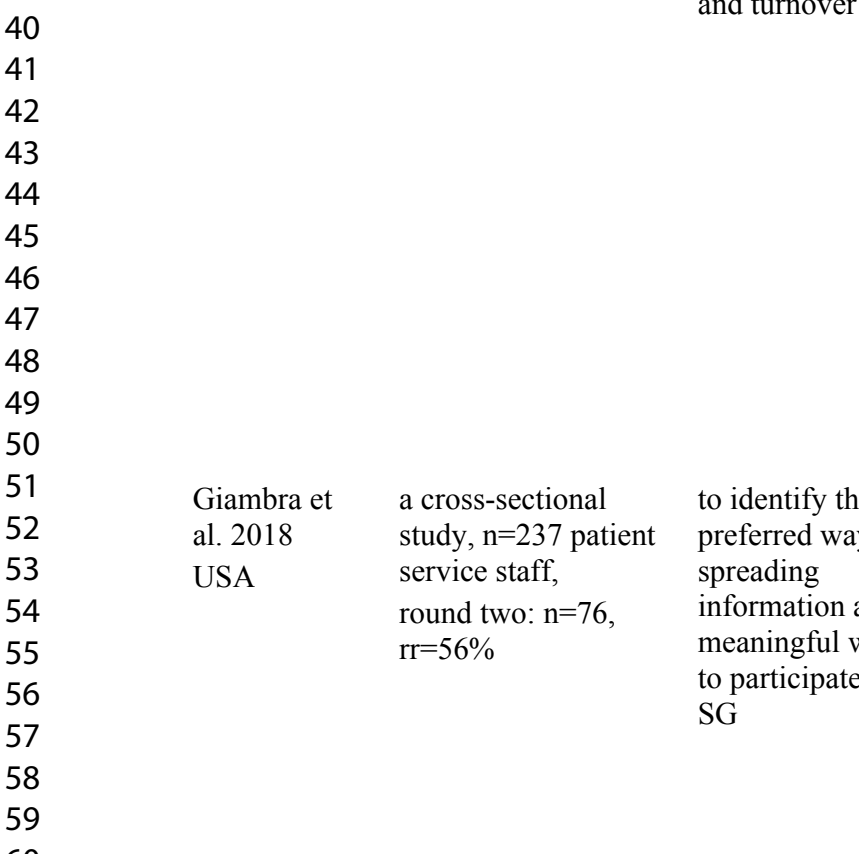

a survey using

the Nursing

Work Index-

Revised, Shared

Governance

Survey, Index of

Work

Satisfaction,

Work Practice

Breakdown

Survey and

Developing

Evidence-Based

Practice as

survey

instruments,

statistical

analysis,

including

descriptive

statistics, a

correlation

matrix and

multiple linear

regressions

a survey using

the Decisional

Involvement

Scale and

statistical

analysis

(calculation of

dissonance

levels)

a survey using

the Work and

Wellbeing

Survey and the

Utrecht Work

Engagement

Scale,

a semi-

structured

interview,

a free textual

and statistical

analysis,

including a

paired t-test and

descriptive

statistics

a modified

Delphi technique

with two rounds

of structured

surveys,

content and

statistical

analysis,

including

descriptive

statistics organizatio all instruments

n-wide indicated

councils improvement

that are except in the final

partially survey year

interdiscipl

inary

unit level

and

organizatio

n-wide

councils

nurses would have

preferred more

control over

decision-making a medical

intensive

care unit

with unit-

level SG

interdiscipl

inary SG

model with

councils at

multiple

levels the survey showed no statistically significant improvements; the focus groups revealed an increase in engagement postintervention, but there was no statistically significant increase in vigour, dedication, or absorption

manager support, scheduled time and adequate coverage for patient care were considered important facilitators of SG participation; communication of ideas from 


\begin{tabular}{|c|c|c|c|c|c|}
\hline $\begin{array}{l}\text { Weaver et al. } \\
2018 \\
\text { USA }\end{array}$ & $\begin{array}{l}\text { a longitudinal study } \\
\text { in two-year } \\
\text { intervals, } n=469 \\
\text { nurses } \\
\text { second round: } \\
n=326 \\
\text { third round: } n=599\end{array}$ & $\begin{array}{l}\text { to identify the } \\
\text { strengths and } \\
\text { weaknesses of the } \\
\text { SG model as well } \\
\text { as the } \\
\text { opportunities it } \\
\text { presents and } \\
\text { threats it faces, } \\
\text { and after the } \\
\text { second round, to } \\
\text { evaluate the } \\
\text { redesigned SG }\end{array}$ & $\begin{array}{l}\text { surveys using } \\
\text { IPNG } 2.0 \text { and } \\
3.0 \text {, } \\
\text { statistical } \\
\text { analysis, } \\
\text { including } \\
\text { descriptive } \\
\text { statistics }\end{array}$ & $\begin{array}{l}\text { unit level } \\
\text { and } \\
\text { organizatio } \\
\text { n-wide } \\
\text { councils }\end{array}$ & $\begin{array}{l}\text { first and second } \\
\text { round evaluations } \\
\text { placed the } \\
\text { organization in the } \\
\text { traditional } \\
\text { governance range } \\
\text { but after } \\
\text { redesigning the } \\
\text { model, the } \\
\text { organization } \\
\text { reached the SG } \\
\text { range }\end{array}$ \\
\hline $\begin{array}{l}\text { Di Fiore et } \\
\text { al. } 2018 \\
\text { USA }\end{array}$ & $\begin{array}{l}\text { a longitudinal study, } \\
\mathrm{n}=106 \mathrm{RNs}, \mathrm{rr}=14 \\
\text { round two: } \mathrm{n}=197 \\
\mathrm{rr}=27 \%\end{array}$ & $\begin{array}{l}\text { to examine nurses' } \\
\text { perceptions of } \\
\text { shared decision- } \\
\text { making } \\
\text { immediately after } \\
\text { implementing SG } \\
\text { and } 3 \text { years } \\
\text { afterwards }\end{array}$ & $\begin{array}{l}\text { a survey using } \\
\text { IPNG and } \\
\text { statistical } \\
\text { analysis, } \\
\text { including a } \\
\text { multivariable } \\
\text { linear regression } \\
\text { model }\end{array}$ & $\begin{array}{l}\text { unit level } \\
\text { and } \\
\text { organizatio } \\
\text { n-wide } \\
\text { councils as } \\
\text { well as a } \\
\text { nursing } \\
\text { congress } \\
\text { (council) }\end{array}$ & $\begin{array}{l}\text { the governance } \\
\text { was perceived to } \\
\text { be primarily } \\
\text { completed by } \\
\text { nurse managers } \\
\text { and the IPNG } \\
\text { score improved } \\
\text { only slightly in the } \\
\text { second round }\end{array}$ \\
\hline
\end{tabular}

* $\mathrm{RN}=$ registered nurse, ${ }^{* * \mathrm{SG}}=$ shared governance, ${ }^{* * *} \mathrm{IPNG}=$ The Index of Professional Nursing Governance 


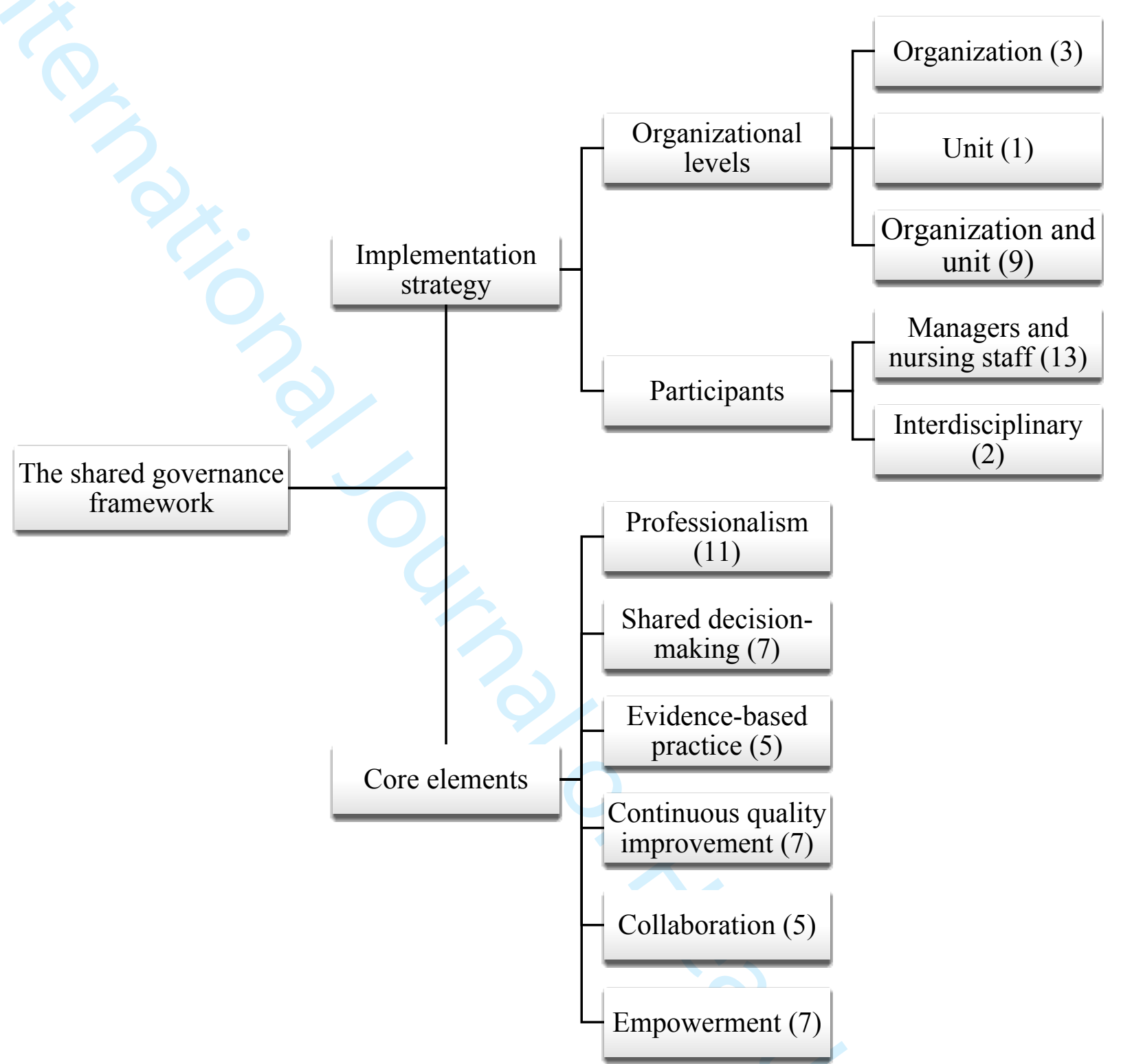

Figure 2. The shared governance framework presented as a map of outcomes. Numbers in parentheses indicate the number of articles included in the scoping review that discussed the corresponding elements and types of implementation strategy. 